\title{
Making advanced electrogravimetry as an affordable analytical tool for the battery interface characterization
}

\author{
Pierre Lemaire ${ }^{\dagger, \ddagger, \S}$, Thomas Dargon ${ }^{\dagger}, \S$, Daniel Alves Dalla Corte ${ }^{\dagger, \S}$, Ozlem Sel ${ }^{\prime}, *$, Hubert Perrot ${ }^{\prime}$, \\ Jean-Marie Tarascon ${ }^{\dagger},, *$ \\ + Chimie du Solide et de l'Energie, UMR 8260, Collège de France, 11 Place Marcelin Berthelot, 75231 \\ Paris Cedex 05, France \\ ‡ Sorbonne Université, 4 Place Jussieu, 75005 Paris, France \\ $\S$ Réseau sur le Stockage Electrochimique de l'Energie (RS2E), CNRS FR 3459, 33 Rue Saint Leu, 80039 \\ Amiens Cedex, France \\ ' Sorbonne Université, CNRS, Laboratoire Interfaces et Systèmes Electrochimiques, LISE, UMR 8235, \\ 75005 Paris, France \\ * Corresponding authors \\ Ozlem Sel: ozlem.sel@sorbonne-universite.fr \\ Jean-Marie Tarascon: jean-marie.tarascon@college-de-france.fr
}

\begin{abstract}
Numerous sophisticated diagnostic techniques have been designed to monitor Electrode-Electrolyte Interfaces that mainly govern the lifetime and reliability of batteries. Among them, is the electrochemical quartz crystal microbalance that offers valuable insights of the interfaces once the required conditions of the deposited film in terms of viscoelastic and hydrodynamic properties are fulfilled. Herein, we propose a friendly protocol that enlists the elaboration of a homogeneous deposit by spray coating followed by QCM measurements at multiharmonic frequencies to ensure the film flatness and rigidity for collecting meaningful data. Moreover, for easiness of the measurements, we report the design of a versatile and airtight EQCM cell setup that can be used either with aqueous or non-aqueous electrolytes. We also present, using a model battery material, $\mathrm{LiFePO}_{4}$, how dual frequency and motional resistance monitoring during electrochemical cycling can been used as a wellsuitable indicator for achieving reliable and reproducible electrogravimetric measurements. We demonstrate through this study the essential role of the solvent assisting the lithium ion insertion at the $\mathrm{LiFePO}_{4}$ interface with a major outcome being the solvent dependent interfacial behavior. Namely in aqueous media, we prove a near surface desolvation of lithium ions from its water solvation shell as compared with organic molecules. This spatial dissimilarity leads to a smoother Li-ion transport across the LFP- $\mathrm{H}_{2} \mathrm{O}$ interface, hence accounting for the difference in rate capability of LFP in the respective electrolytes. Overall, we hope our analytical insights on interface mechanisms will help in a wider acceptance of EQCM-based methods by the battery community.
\end{abstract}

\section{Keywords}

Battery, Electrode-Electrolyte Interface, Interface characterization, EQCM, Cell development, $\mathrm{LiFePO}_{4}$ 


\section{Introduction}

For the last decades, the Li-ion battery (LIB) chemistry has widely conquered the portable electronics field and is nowadays expanding to the electric vehicle market. Furthermore, batteries seems to be the electrochemical energy storage technology of choice to regulate the renewable energy intermittency for grid application. ${ }^{1}$ Since then, the focus has been placed on finding new materials, improving the intrinsic performances of electrodes to obtain better energy and power densities. ${ }^{2}$ For the purpose of designing materials with interesting electrochemical properties, powerful diagnostic techniques have been developed. Those operando methods like synchrotron X-ray/Neutron diffraction or X-ray absorption spectroscopy permit a better understanding of the fundamental interplays between the long and short range structural evolution and the lithium insertion/extraction process. ${ }^{3,4}$ However, of paramount importance to enhance battery lifetime and reliability is to explore the essential role played by the Electrode-Electrolyte interface (EEI) and its impact on the physicochemical properties of ion intercalation. The vicinity of this intimate interface, has been demonstrated to control long term and kinetic performances of LIBs through important electrode processes, such as the formation of the Solid Electrolyte Interphase ${ }^{5}$ (SEI) which is intimately correlated to electrode stability and possible desolvation process of cations at the electrode interface. Moreover, its dynamic nature upon cycling is a nightmare to electrochemists. Gravimetric-based analytical techniques like Electrochemical Quartz Crystal Microbalance (EQCM) have shown a remarkable sensitivity to get a better insight on those interfacial processes.

Real-time assessment of mass variation, thanks to the well-known Sauerbrey equation ${ }^{6}$, during an electrochemical process as the identification of the products by classical EQCM was firstly introduced by Aurbach et al. in 19957. By studying the surface film formation over a gold electrode in $\mathrm{LiPF}_{6}$ and $\mathrm{LiAs}_{6}$ in propylene carbonate (PC) solvent, the authors have proved a potential-dependent formation mechanism of the SEI. This work got worldwide resonance and studies on SEI have moved to systems that are more realistic enlisting PVD-prepared carbon ${ }^{8,9}$, electrodeposited Sn film ${ }^{10}$ and more recently to the study of drop cast graphite ${ }^{11}$ on the surface of EQCM resonators. Further exploring this technique, our group demonstrated the formation of a F-based passivating layer at the negative electrode surface triggered by the water reduction in the fashionable "Water-in-Salt" electrolyte system. ${ }^{12}$ Apart from SEI, EQCM studies demonstrated that $\mathrm{LiBF}_{4}$ used as an additive can hinder the Aluminum current collector corrosion upon cycling. Some active electrode materials $\mathrm{LiMn}_{2} \mathrm{O}_{4}{ }^{13}, \mathrm{~V}_{2} \mathrm{O}_{5}{ }^{14}$, $\mathrm{WO}_{3}{ }^{15,16}$ or $\mathrm{Nb}_{2} \mathrm{O}_{5}{ }^{17}$ have also been deposited on the surface of EQCM resonators to obtain a better understanding of Li ions insertion/extraction during the charge/discharge process, namely dealing with the reversibility of the mass changes in electrochemical processes and the possible co-intercalation of solvent molecules. In light of such studies, the feasibility of interacting magnesium ions into $\mathrm{MnO}_{2}$ mediated by the shielding of magnesium charge by water molecules in organic electrolyte was proposed. ${ }^{18} \mathrm{An}$ analogous phenomenon has recently been identified during protons insertion in $\mathrm{H}_{\mathrm{x}} \mathrm{IrO} \mathrm{O}_{4}$ in aqueous electrolytes. ${ }^{19}$

The use of EQCM solely as a gravimetric probe implies that resonant frequency changes $(\Delta f)$ caused by the electrochemical processes between the solid surface and the liquid electrolyte are exclusively related to mass changes while no energy is dissipated during the oscillations. ${ }^{20}$ Such assumption requires a judicious analysis of the resonant frequency changes $(\Delta f)$, enabling their assignment to true processes. Ideally, a dense, flat and thin layer of active material is rigidly attached to the resonator and therefore follows synchronously its motion without dissipation of energy. Practically, such conditions are not easily achieved in case of composite electrodes glued together by polymeric chains (binders) and presenting different porous geometries. This also applies to the SEI formation where the grown layer is composed of several inorganic and organic constituents. To overcome such difficulties, the so- 
called multiharmonic EQCM-D technique that conjugates $\Delta f$ measurements with dissipation $(D)$ energy monitoring at different harmonics $(n)$ of the quartz fundamental resonant frequency has been proposed. This powerful technique can be viewed as structural/morphological and mechanical/viscoelastic probe to study non-ideal films consisting of porous electrodes and binders by taking into account of non-gravimetric hydrodynamic and viscoelastic effects that cannot be handled by EQCM alone. Pioneered by Levi and Aurbach, EQCM-D is getting momentum within the field of batteries to screen electrolytes, additives for achieving homogeneous and thin SEl. ${ }^{21,22}$ Innovative approaches combining EQCM-D with Online Electrochemical Mass Spectrometry (OEMS) have also being reported to grasp insights into the SEI formation mechanism on both model and practical electrodes. ${ }^{23,24}$ Last, and of paramount importance has been the use of EQCM-D to understand the binder-particles ${ }^{25,26}$ and solvent-binder ${ }^{25,27-29}$ interplays within the electrode as well. Altogether, these shows the span covered by EQCM-D to the battery field and exhaustive list can be found in references. ${ }^{20,30,31}$

Although quite elegant, this technique is not free of improvements that can range from simplifying its mathematical aspects to make it more comprehensive, more user-friendly and more compatible with today battery tooling. Searching for a compromise, we herein propose a derived technique called $E Q C M-R$, which conjugates the monitoring of the resonance frequency change and of the motional resistance $(R)$. Compared with multiharmonic EQCM-D, EQCM-R compromises the advantage of the former to the benefit of cheaper and more stable measurements. ${ }^{32,33}$ Moreover, rather than measuring quantitatively the viscoelastic properties of the deposited composite electrode upon cycling, EQCM-R assesses the motional resistance as a viscoelastic indicator. Last and of paramount importance regardless the technique considered (EQCM, multiharmonic EQCM-D or proposed EQCM-R) is to define a proper analytical approach, suitable to the field of study (i.e. here for batteries).

To address these issues and to widen the spectrum of EQCM measurements, we propose herein a strategy that combines thin film preparation on QCM specific substrate, design of EQCM cells, and definite measurements protocols. This includes an optimized spray coating technique to elaborate routinely and reliably composite electrode deposits at the surface of EQCM resonators, with the technique being tunable according to the physicochemical properties of the components (active material, binder, etc.). A major development is on the design of hermetically sealed and versatile EQCM cell to mimic battery conditions that can be used either with aqueous or non-aqueous electrolytes. Moreover, our designed EQCM cell can be coupled to lab-made and commercial microbalances to assess the nature of the electrode material (stiff, soft) and its interaction with the electrolyte. For proof of concept, we demonstrated the reliability and reproducibility of our electrochemical and gravimetric measurements made by EQCM-R on a model battery material, $\mathrm{LiFePO}_{4}$.

To convey such a new tooling and strategy, our paper will be structured as follows. The importance of mastering the thin film deposition on the QCM substrate is first described prior to addressing the design of new EQCM cells for operating in both aqueous and organic media. Secondly, we present how to ensure conformity of the hydrodynamic and viscoelastic properties of the coating with gravimetric regime requirements prior to showing the implementation into the EQCM-R in studying model $\mathrm{LiFePO}_{4}$ electrodes. Lastly, the benefits of both the proposed methodology and new tooling are discussed with respect to the state of the art of the field. 


\section{Experimental section}

Electrode preparation. AT-cut $9 \mathrm{MHz}$ quartz resonators patterned with gold electrodes on both sides were used as EQCM electrodes $\left(0.196 \mathrm{~cm}^{2}\right)$. Lithium Iron Phosphate $\mathrm{LiFePO}_{4}$ was mixed with $18 \mathrm{wt} \%$ Csp and $10 \mathrm{wt} \%$ PVDF-HFP in 1-Methyl-2-pyrrolidinone by ultrasonication to obtain a homogenous slurry with different concentrations. Then a $300 \mu \mathrm{L}$ volume of slurry was spray casted with an airbrush or drop casted on the gold electrode of the quartz resonators. The electrode loading was obtained thanks to the resonant frequency change.

Airtight EQCM cell testing workbench. A bare quartz resonator was mounted between two perfluoro elastomer O-rings known for their non-swelling behavior in organic electrolyte. Then it was inserted in a labmade cell that we designed specifically for EQCM measurements with its unique role of being used either with aqueous or organic electrolyte and which was developed conjointly by CSE lab and Sphere ENERGY. The cell airtightness was evaluated thanks to water uptake quantification by Karl Fischer titration. The cell impedance was also measured by impedance spectroscopy.

Verification of hydrodynamic viscoelastic properties in electrolyte. Quartz crystal microbalance with dissipation monitoring was performed using AWS A20 (Advanced Wave Sensors) from the 3rd to the 11th overtone in order to check the hydrodynamic and viscoelastic properties of the spray casted film and verify the gravimetric regime on Au-coated resonator with fundamental frequency of $9 \mathrm{MHz}$. The resonant frequency, $f$, and the absolute dissipation factor, $D$, was recorded at the different overtones during the passage from air to the electrolyte solution either $1 \mathrm{~mol} / \mathrm{L} \mathrm{LiClO}_{4}$ in Milli-Q ultrapure water or propylene carbonate under Open-Circuit Potential (OCP).

Electrochemical-gravimetric analysis. Specifically designed EQCM cell described above coupled with a commercial SEIKO QCM922A microbalance was employed to monitor the resonance frequency change along with the motional resistance $(R)(E Q C M-R)$ during the electrochemical processes. The LFP composite electrode was cycled in $1 \mathrm{~mol} / \mathrm{L} \mathrm{LiClO}_{4}$ aqueous and organic electrolyte, Milli-Q ultrapure water and propylene carbonate respectively. For both media, the EQCM electrode was used as the working electrode. For aqueous electrolyte, a $3 \mathrm{~mm}$ diameter graphite rod (Sigma-Aldrich) as the counter electrode and $\mathrm{Hg} / \mathrm{Hg}_{2} \mathrm{SO}_{4}$ (saturated $\mathrm{K}_{2} \mathrm{SO}_{4}$ ) as the reference electrode are used. Whereas for organic electrolyte, lithium metal disks of 5 and $2 \mathrm{~mm}$ were punched to be used as counter and reference electrodes, respectively. During the electrochemical cycling, the quartz resonant frequency and motional resistance were simultaneously measured. Simultaneous electrochemical measurements were performed using a SP-200 potentiostat (Bio-Logic). A more detail experimental section is contained in the Supplementary information. 


\section{Results}

Optimization of the electrode preparation. Previous EQCM-D studies clearly indicate that when the electrode surface is rough or the electrode itself is porous, additional contribution to frequency changes may occur depending on the pore width, i.e. the hydrodynamic effect of the trapped or mobile liquid in the pores ${ }^{20,31}$. Therefore, the deposition of the composite layer without lateral or vertical inhomogeneity should be one of the primary concern in the good practice of the EQCM analyses. Guided by this idea, a considerable amount of effort has been devoted in our study towards the development of the most suitable protocol leading to a homogeneous film rigidly attached on the surface of the QCM resonator. To this end, a deposition process based on spray coating was elaborated and the electrodes were treated under "soft conditions" without perturbing the quartz resonators piezoelectric properties.

To mimic the electrode used in practical batteries, composite electrodes made of an active material, a conductive agent and a binder were targeted. For this specific study, commercial LiFePO $_{4}$ having submicrometer particles $(\simeq 200 \mathrm{~nm}$ ) were employed as active material to obtain a thin, compact and multi-layered coating. Polyvinylidene fluoride (PVDF) has been chosen as a binder because of its high chemical inertness, its good mechanical properties and its less hydrophobic nature compared to Polytetrafluoroethylene (PTFE) for aqueous cycling. To facilitate the composite film preparation under softer conditions, the copolymer Poly(vinylidene fluoride)-co-hexafluoropropylene (PVDF-HFP) was selected due to its lower melting temperature compared to that of classical PVDF. The outlines of the protocol are depicted in the Figure 1.

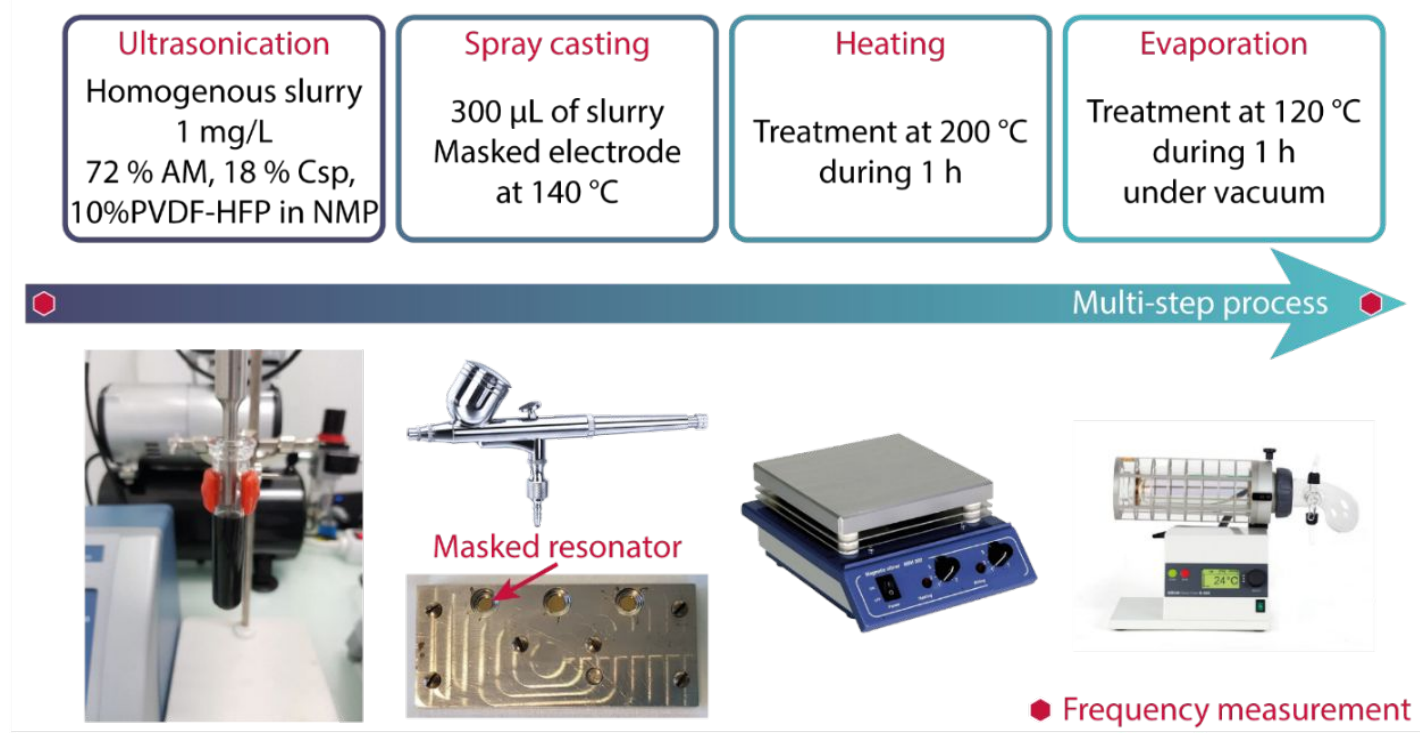

Figure 1: Outlines of the different steps permitting a composite electrode on the surface of a QCM resonator to be obtained. Further experimental details are in the Supplementary Information Part I.

However, the fine-tuning of the process is rooted in mastering a few details through the various steps. The ultrasonication step for instance permits to obtain a homogeneous slurry with different PVDFHFP/LFP weight concentrations. The combination of the proper heating temperature and the small droplets created by the spray enables the fast evaporation of the solvent. Here, air has been used as the spray gas vector but it can be replaced by argon or nitrogen depending upon the nature of the active material. At this stage, for the sake of comparison, exactly the same protocol was applied by replacing the spray coating by the classical drop casting. The Figure S1 shows the morphology differences between the coatings obtained by those two deposition techniques that is in favor of spray 
coating. The drop casting demonstrates the coffee-ring effect ${ }^{34}$ compared to the macroscopic homogeneous layer obtained by the spray coating method. However, at the microscopic scale, agglomerates of materials can be found at the surface of the electrode (Figure S2a). To circumvent this issue, the film is exposed to a heating treatment at $200{ }^{\circ} \mathrm{C}$ to enable the spreading of the PVDF-HFP thermoplastic binder $\left(\mathrm{T}_{\text {melting }}=150{ }^{\circ} \mathrm{C}^{35}\right)$ and minimizing its coating roughness so that a laterally homogeneous and flat film as revealed by SEM images (Figure S2b) is observed. Such a heating treatment should not affect the resonator properties, which are reported to be maintained until 573 ${ }^{\circ} \mathrm{C}$ at which the $\alpha-\beta$ phase transition in quartz occurs. ${ }^{36}$ In addition, the resonant frequency of a bare quartz, like the motional resistance, remains constant before and after the heating treatment. The resonant frequencies of the bare and loaded resonator were measured in air at room temperature to obtain the frequency change and the expected mass of the composite electrode thanks to the Sauerbrey equation ${ }^{6}((1)$ and therefore the loading. The same measurements were done to get the motional resistance $(R)$ change of the resonator after the coating.

$$
\Delta m=-C_{f} \cdot \Delta f
$$

Putting aside the macroscopic aspect of the composite electrode, its frequency change and motional resistance $(R)$ obtained by spray coating and drop casting have been compared as a function of slurry concentration in material as depicted in Figure 2. It is noted that the resonators have been prepared using rigorously the same conditions, i.e. especially the cast and sprayed volume of $300 \mu \mathrm{L}$. The Figure $2 \mathrm{a}$ shows the as expected quasi-linear behavior between the frequency change and the slurry concentration for the spray coating method. As for the drop casting, it demonstrates a linear behavior for concentration below $1 \mathrm{mg} / \mathrm{mL}$ then the frequency change seems to stagnate. In the linear region, for the same concentration, the frequency changes obtained by spray coating are lower than by drop casting. Consequently, based on the $\mathrm{LiFePO}_{4}$ sample specimen used, the spray coating is a more suitable option to obtain a wide range of predictable frequency change, therefore loading and the presented figure can be used as a calibration curve (Figure 2a). 


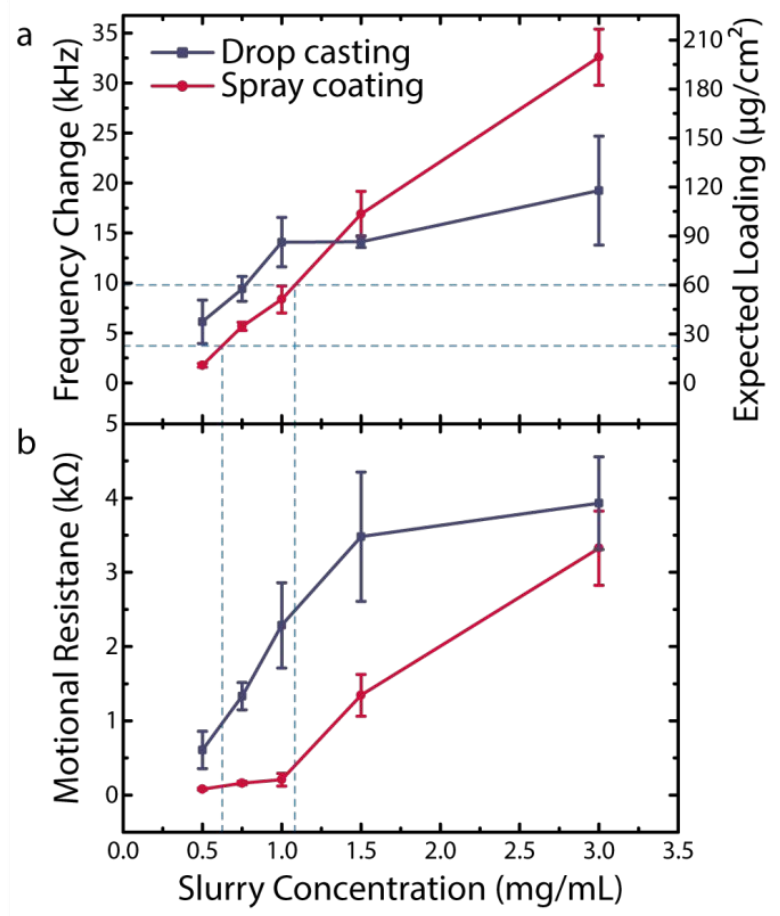

Figure 2: (a) Frequency changes, loadings and (b) motional resistances of composite films measured in air prepared by drop casting or spray coating techniques as a function of the slurry concentration (the cast and sprayed volume are kept at a constant value of $300 \mu \mathrm{L}$ ).

The motional resistance $(R)$ values are used to evaluate the coating quality obtained by the two methods. The $R$ for the drop cast coatings (Figure 2b) shows a linear behavior as the slurry concentration increases and a plateau at values exceeding $1.5 \mathrm{mg} / \mathrm{mL}$. This saturation coincides roughly with frequency change saturation observed in Figure 2a. Significantly lower $R$ values were observed for the spray coated resonators, however they also exhibit a threshold and substantially increase for concentration values higher than $1 \mathrm{mg} / \mathrm{mL}$. Considering the high $R$ values for high slurry concentrations, we can assert the deviation from the gravimetric conditions, especially significant for drop-cast coatings. Therefore, the respective loadings given at the high end of the Figure 2a are only estimated and may deviate from the genuine values, due to the energy dissipation. However, even when the loading is higher, the spray coating results in relatively lower motional resistance. Before the threshold, the $R$ values are low $(<150 \Omega$ ) compared to that of the bare electrode $(10 \Omega)$. Smaller error bars in the case of spray coating are also noted. The full resonance width change $(\Delta W)$ was also calculated thanks to a procedure described in the Supplementary information. The condition of the gravimetric sensing, i.e. $|\Delta W| \ll|\Delta f|$ was found only in the case of spray coating for slurry concentration strictly below $1.5 \mathrm{mg} / \mathrm{mL}$ (Figure S3), for the electrode composition of this study. Therefore, the spray coating has been selected to obtain composite LFP films with higher reproducibility and lower motional resistance. A slurry concentration of $1 \mathrm{mg} / \mathrm{mL}$ has been chosen as uppermost point to obtain loadings in a range between 20 and $60 \mu \mathrm{g} / \mathrm{cm}^{2}$ and employed in the electrogravimetric investigations, thereafter. It is noted that these conditions are specific to LFP nanoparticles and the PVDF-HFP binder employed here and should be redefined using the methodology described above depending on the active material and/or binder.

Design and validation of airtight EQCM cell testing workbench. Past and present cells to perform EQCM measurements suffer among others of lack of airtightness, incompatibility with battery-like architecture (small volume of electrolyte, electrode geometry and positioning) and/or a cumbersome assembly. These drawbacks can hinder the wider exploitation of EQCM in the battery field. Therefore, 
we designed and built a dedicated tooling with required cell characteristics. Specific cell designs are represented in the Figure 3. The assembly is convenient to be manipulated in a glovebox, during the loading of air sensitive components into the cell (e.g. metallic Li electrodes, electrolytes, etc.), but it is designed to be used for performing electrochemical tests outside the glovebox thanks to its hermetic closing mechanism. Polypropylene (PP) has been selected as the core cell material because of its numerous advantages: good resistance to fatigue, chemical resistance to almost all organic solvents, low density and hydrophobic property, which makes it easier to dry when switching from aqueous to organic electrolytes. Within this new design, the oscillator is connected to the cell thanks to a BNC connector as represented in the Figure S4 and the potentiostat via $2 \mathrm{~mm}$ banana plugs to obtain reliable connections. Using such standard connections, this cell can be easily interrogated by different lab-made or commercial methods of read-out: EQCM, EQCM-R or Network analyzer and it can be made compatible with available EQCM-D apparatus. The cell impedance, as deduced by impedance spectroscopy (Figure S5), presents a negative straight-line characteristic of an inductance in series with a resistance which values were calculated as $308 \mathrm{nH}$ and $35.6 \mathrm{~m} \Omega$, respectively. The smallness of these values demonstrates only minor influence of the cell impedance to the electrochemical impedance spectroscopy (EIS) measurements, which expands the versatility of EQCM cell to other coupled methods, e.g. QCM coupled to EIS, the so-called ac-electrogravimetry. ${ }^{37}$

$a$

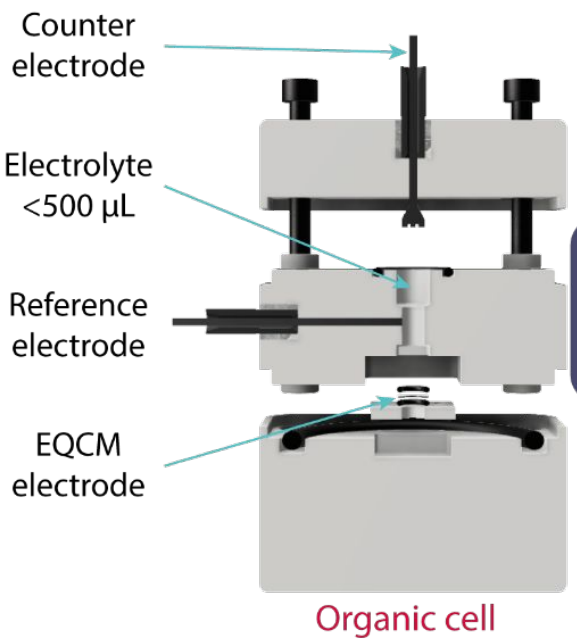

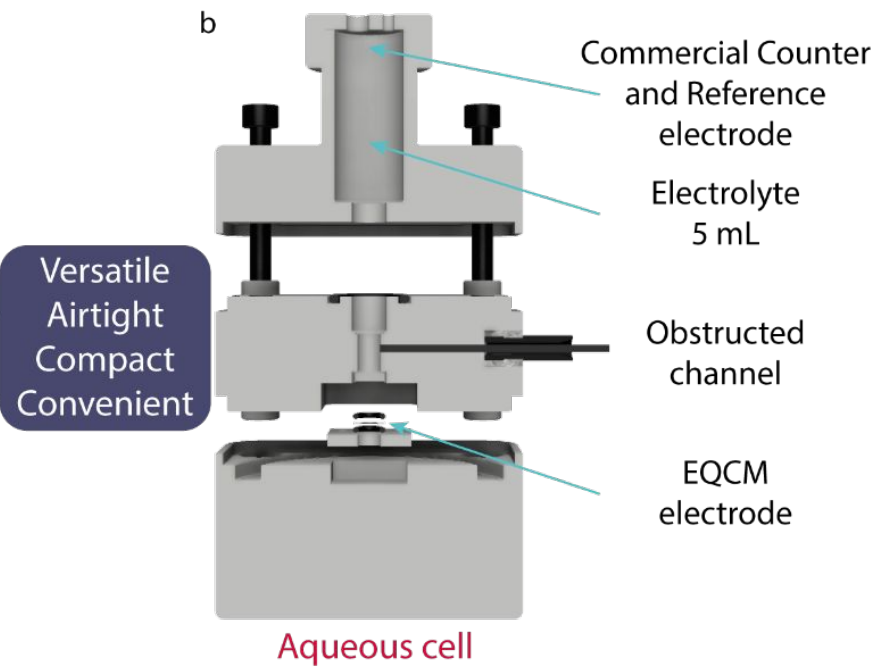

Figure 3: Graphical representation of the EQCM cell designed for battery electrode testing in (a) organic and (b) aqueous electrolytes.

The required specifications for development of an EQCM cell hosting an organic electrolyte were to mimic the configuration used in a practical Li-based battery. Therefore, the counter electrode is facing the resonator with the same surface. The distance between them has been minimized to obtain a volume of electrolyte comprised between 300 and $500 \mu \mathrm{L}$. Additionally, the cell can host a reference electrode through a lateral opening. In this work, we used a piece of lithium metal placed at the tip of a stainless steel rod as reference electrode to obtain a more realistic measurement of the different potentials, working and counter electrodes. To avoid leakage of the electrolyte, perfluoro elastomer O-rings known for their non-swelling behavior in organic electrolyte were used in conjunction with four screws to impose pressure and provide the complete airtightness to the cell. The efficacy of the cell against water uptake upon time has been monitored by Karl Fischer titration (Figure S6) and after two weeks, a water contamination by less than $60 \mathrm{ppm}$ was measured. Therefore, the electrochemical measurements in organic electrolyte can be performed in a non-inert atmosphere without the risk of water or oxygen contamination. Moreover, this EQCM cell has a compact geometry that facilitates the 
transfer in and out of the glovebox through a small antechamber. Last and worth mentioning is the versatility of our cell setup that enables to perform battery testing in aqueous electrolyte by replacing the top part of the cell to increase the electrolyte volume for enabling the use of commercial counter and reference electrodes (aqueous-based).

At this stage, it was mandatory to check any eventual perturbation of the quartz resonant frequency by our new cell confinement. Bearing in mind that AT-cut quartz crystals are typically used due to their low sensitivity to temperature ${ }^{38}$, we have inspected the temperature dependence of the resonant frequency, $f$, in this specific cell design. Intervals of different temperature has been applied to the EQCM cell equipped with a bare resonator and its $\Delta f$ has been monitored over time (Figure S7). A drastic decrease of the $f$ appears synchronously with the temperature rise. However, the $f$ stabilization is much slower than that of temperature. Thus, the temperature is an important factor to take into account during long cycling and makes $f$ measurement practically incompatible with glovebox conditions. Therefore, we recommend to launch experiments after sitting the EQCM cell at Open-Circuit Potential (OCP) for $4 \mathrm{~h}$ to obtain the frequency stability before performing battery cycling tests at $25{ }^{\circ} \mathrm{C}$. Pleasantly, it can be noted that the frequency change is totally reversible with the temperature.

The sensitivity factor $\left(C_{f}\right)$, that is also an essential input parameter, has been estimated thanks to the classical $\mathrm{Cu}$ electrodeposition. For the sake of comparison, both galvanostatic electrogeneration and cyclic voltammetry were employed and the results are presented in the Figure $\mathrm{S} 8$. The slope between the $\Delta f$ and the $\Delta m$ (estimated by the Faraday's law, Equation S2) provides the experimental $C_{f}$ value in (1. To obtain good statistics, the experiments were performed several times with different applied current densities and scan rates (Table S1), leading to the experimental $C_{f}$ value of $1.21 \pm 0.03 \mathrm{ng} / \mathrm{Hz}$ that is used for the electrogravimetric studies, thereafter.

Lastly, the motional resistance $(R)$ during the electrodeposition/stripping of copper was also recorded and it is plotted in the Figure S9. We measured a $\Delta R$ of $25 \Omega$ for the electrodeposition by galvanostatic method, while the cyclic voltammetry demonstrates a reversible variation of solely $10 \Omega$. Both processes (Figure $\mathrm{S} 8$ and $\mathrm{S} 9$ ) lead to the high $\Delta f / \Delta R$ ratios, indicator for the rigidity of the metallic layer. ${ }^{39} \mathrm{~A}$ slightly higher $\Delta f / \Delta R$ ratio is noticed for the film obtained by $\mathrm{CV}$, which can be explained by an electrodeposition method-dependent morphology leading to slight difference in hydrodynamic properties.

Verification of hydrodynamic and viscoelastic properties in electrolyte. At this stage, by combining optimized coating process and the new cell design, we have made part of the requirements towards the development of an EQCM based research tool compatible with battery testing. However, to conveniently interpret the EQCM results into exploitable mass change, another important aspect has to be checked. When the coating is transferred to electrolyte, its hydrodynamic and viscoelastic properties need to remain negligible, so that they do not intervene to the microbalance functioning. The optimized coating procedure of our work is expected to minimize the lateral inhomogeneity of the LFP composite film and thus, minimize the oscillation energy dissipation originated from the rough surface/electrolyte interactions. However, for a definite validation, QCM with dissipation monitoring was performed following the protocol developed by the group of Levi and Aurbach. ${ }^{31}$ The resonant frequency, $f$, and the absolute dissipation factor, $D$, was recorded at the different overtone orders, $n$ during the passage from air to the electrolyte solution ( $1 \mathrm{~mol} / \mathrm{L} \mathrm{LiClO}_{4}$ in water or propylene carbonate) under Open-Circuit Potential (OCP). The absolute dissipation factor has been replaced by the resonant width thanks to the relation: $W=f \times D$. Such measurements are depicted in the Figure S10. Independently of the solvent, $\Delta f / n$ and $\Delta W / n$ demonstrated a non time-dependent behavior and 
moreover decrease algebraically with the overtone order. The steadiness of the signals at each overtones manifests the rigidity of the film and the rigid bonding between the coating and the quartz, in agreement with the previous studies. ${ }^{28,29,31}$ To obtain further insights on the solid-liquid interaction (the hydrodynamic properties), $\Delta f / n$ and $\Delta W / 2 n$ normalized by $\rho_{l} f_{0}^{2}$ (where $\rho_{l}$ and $\eta_{l}$ are the density and the dynamic viscosity of the studied liquid) are plotted as a function of the penetration depth of the shear wave, $\delta$, across the boundary layer between the resonator and the electrolyte in the Figure 4.

$$
\delta=\sqrt{\frac{\eta_{l}}{\pi n f_{0} \rho_{l}}}
$$

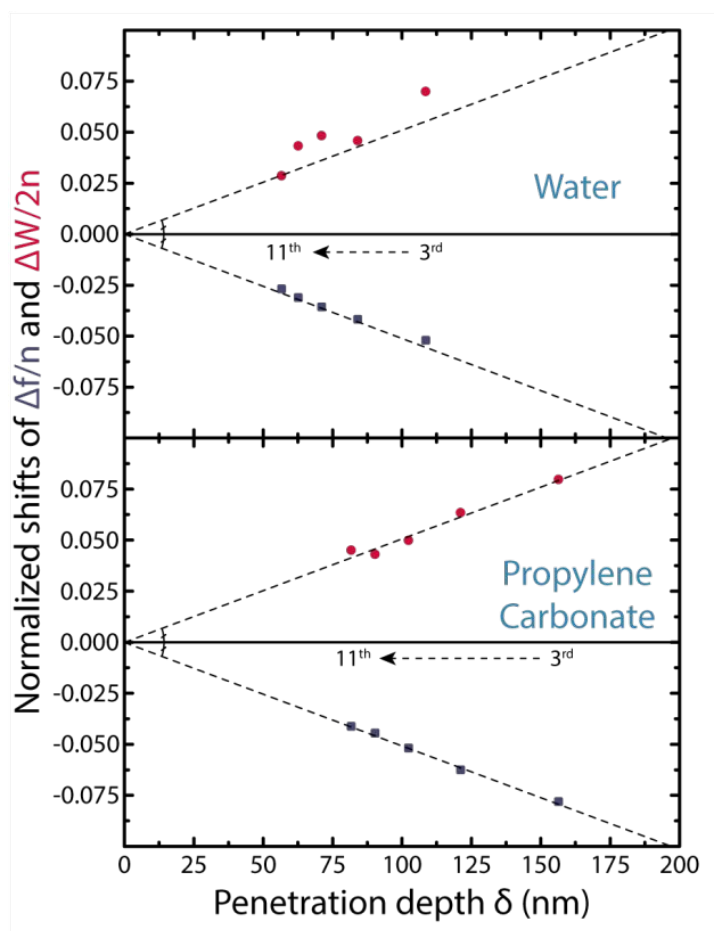

Figure 4: Normalized (by overtone order as well as by $\rho_{l} f_{0}^{2}$ ) shifts of frequency $\Delta f / n$ (blue) and resonance width $\Delta W / 2 n$ (red) as a function of the penetration depth. Shifts are referenced to that of the film in air rather than to the bare crystal. The top (bottom) panel represents the measurement performed in water (propylene carbonate).

This figure shows that the penetration depth values are smaller in the case of aqueous electrolyte due to the lower density of water. QCM-D is therefore more sensitive in aqueous than in organic electrolyte, which may explain the slightly more scattered $\Delta W / 2 n$ data, for the former. In both solvents, $\Delta f / n$ and $\Delta W / 2 n$ versus $\delta$ data present straight lines with a quite similar slope (in absolute value). The modified Kanazawa and Gordon equation, ${ }^{40}$ which describes the hydrodynamic interaction between a film with a flat surface and a Newtonian liquid leads the normalized frequency and resonance width shifts to solely depend on the penetration depth, $\delta$ according to the following equations: ${ }^{31,41}$

$$
\frac{\Delta f}{n \rho_{l} f_{0}^{2}}=-\frac{\delta}{\sqrt{\mu_{q} \rho_{q}}} \quad \frac{\Delta W}{2 n \rho_{l} f_{0}^{2}}=\frac{\delta}{\sqrt{\mu_{q} \rho_{q}}}
$$

Further details about the equation demonstration are given in the Supplementary Information Part IV. These two values leads to a straight line as a function of the penetration depth of which slope only 
depends on the intrinsic properties of the quartz, its density $\rho_{q}$ and shear modulus $\mu_{q}$. Here, the straight lines with similar slopes depicted in the Figure 4 are QCM-D signature of the hydrodynamic interaction between liquid and stiff solid film with plane surface. ${ }^{20}$ In conclusion, our coating process of the composite LFP film is optimized and fulfills the gravimetric conditions under OCP and therefore, the Sauerbrey relationship can be employed to treat microbalance data.

Electrochemical-gravimetric measurements of an intercalation material. After validation of the metrics regarding the EQCM cell design and mastering the coating attributes, time has come to implement our analytical approach to battery testing. For proof of concept, $\mathrm{LiFePO}_{4}$ is particularly suitable as an active material due to its versatile cyclability both in organic and aqueous electrolytes. As the redox potential nicely falls into the thermodynamic stability of the electrolyte, it eliminates the risks of parasitic reactions ${ }^{42}$ (transition metal dissolution and growth of a Cathode-Electrolyte Interface). Frequency change and absolute motional resistance were simultaneously measured on LFP-based deposits during cyclic voltammetry at various scan rates in water or in propylene carbonate based electrolyte in $1 \mathrm{~mol} / \mathrm{L}$ of $\mathrm{LiClO}_{4}$. The results for each electrolyte and each scan rate are gathered in the Figure 5 . The small variation (less than $2 \%$ ) of the motional resistance in both electrolytes during CV (Figure 5, third row) reveals the rigidity retention ${ }^{43}$ during the lithium insertion/extraction. Such an EQCM-R testing protocol ensures that the Sauerbrey equation applies for extracting the $\Delta m$ from the measured $\Delta f$ values, using (1. In addition, it is reinforced by the small full resonance width changes compared to the frequency changes $(|\Delta W| \ll|\Delta f|)$ during cycling in both electrolyte (Figure S11). As the measurements were not performed on the same electrode with identical loading, the $\Delta m$ has been normalized to be able to compare the electrogravimetric behavior in two different electrolytes. 

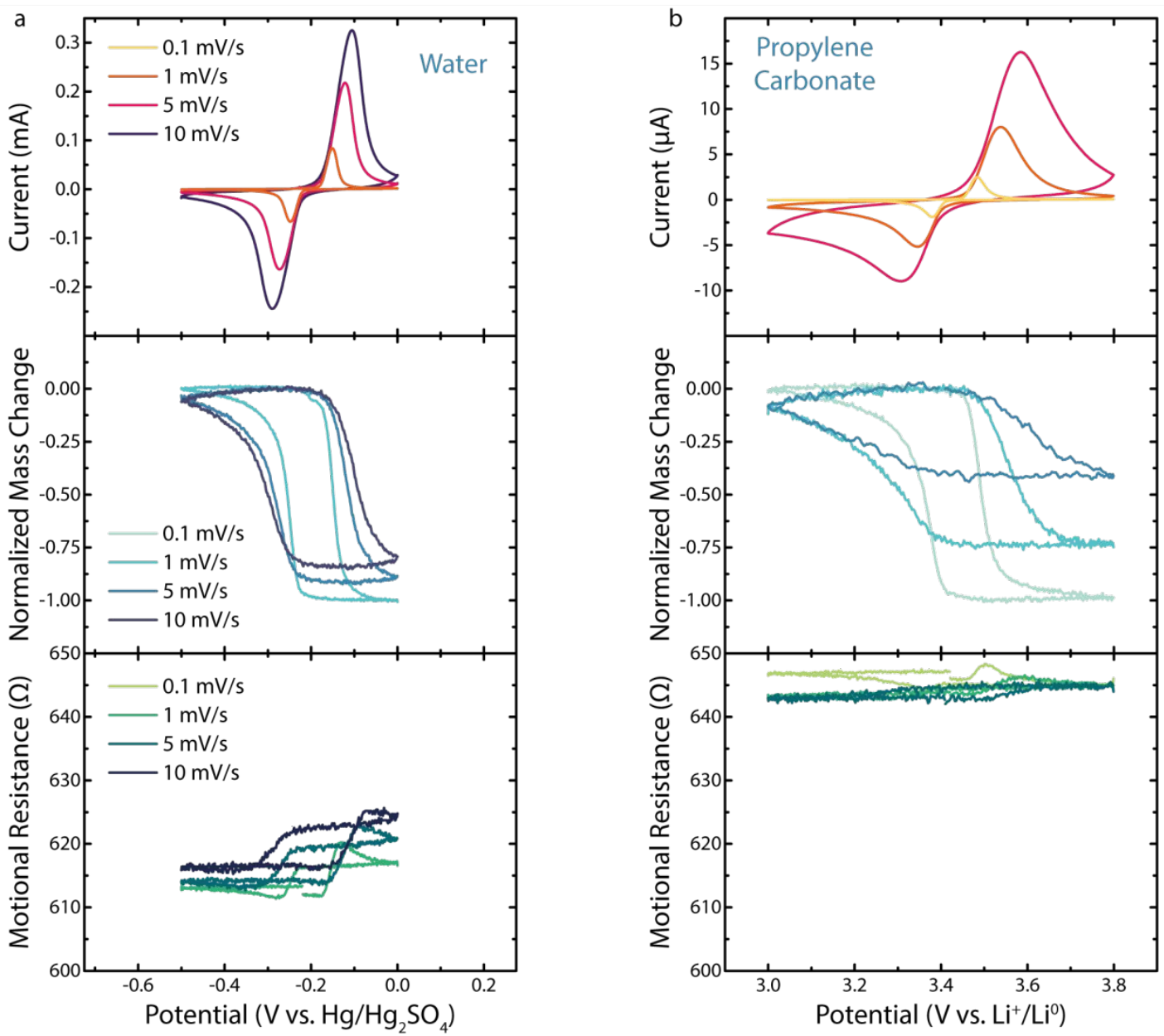

Figure 5: Cyclic voltammetry of LFP composite electrodes at different scan rates, in $1 \mathrm{~mol} / \mathrm{L} \mathrm{LiClO}_{4}($ a) in water and (b) in propylene carbonate. Intensity, frequency change and absolute motional resistance were simultaneously measured. The frequency change was converted into mass change and normalized by the active material loading. The fifth cycle is here represented for each scan rate.

Classical electrochemical response of LFP was observed in both organic and aqueous electrolytes with redox peaks centered around $3.44 \mathrm{~V}$ vs. $\mathrm{Li}^{+} / \mathrm{Li}^{0}$. Aqueous electrolyte presents sharper peaks than organic electrolyte even if it has been performed at faster scan rates. The potential difference of the redox peaks is also smaller in aqueous electrolyte as well as the hysteresis in normalized $\Delta m$ responses. Since this hysteresis is more pronounced in organic electrolyte, the mass variation spreads on a larger potential window. The difference between the maximum and the minimum mass directly reflects the (de)lithiation stoichiometry in $\mathrm{Li}_{x} \mathrm{FePO}_{4}$. Its dependence on the scan rate can be correlated with the dynamic of lithium ions insertion/extraction into the electrode material. In comparison to thick composite electrode used in practical application, it must be stressed that coatings of limited masses provide a facile accessibility of the electrolyte to the intercalation particles. This enables the full lithiation/delithiation occurring in a shorter period of time.

Exploring further the differences between aqueous and non-aqueous electrolytes, we note for the former an increase in the scan rate results in a slight decrease of the normalized $\Delta m$ (Figure $5 a$, middle panel). This contrasts with the organic electrolyte, which shows a substantially smaller normalized $\Delta m$ with increasing scan rate, in an extent that solely half of the particles is delithiated at a $5 \mathrm{mV} / \mathrm{s} \mathrm{scan}$ rate (Figure $5 \mathrm{~b}$, middle panel). Therefore, electrogravimetric measurements clearly prove a better rate capability of LFP in aqueous rather than in organic electrolyte. Additionally, by performing 
electrogravimetric analyses on both aqueous and non-aqueous electrolytes over long cycling (more than 50 cycles) we note a sustained synchronization between the redox peaks and the mass variation upon cycling (Figure S12), indicative of the robustness of our measurements. Moreover, we intriguingly observed in both water and organic electrolytes a linear drift of the $Q$ versus time, which cannot be explained by a SEI formation especially in water (Figure S13). This drift was identified as intrinsic to our potentiostat and to all brands, with presence of a brand-dependent slope (Figure S14). Having identified this problem, the $Q$ curve has been corrected by this slope coefficient, as represented in the Figure $\mathrm{S} 13$, before being translated into theoretical $\Delta m_{\text {Faraday }}$.

Next, we compared (Figure 6a) the electrochemical mass variation $\Delta m_{\text {Faraday }}$ associated to the lithium ion insertion/extraction processed from the charge $(Q)$ passing through the system (thanks to the Faraday's law) against the mass variation $\Delta m_{E Q C M}$ estimated by the Sauerbrey equation. A striking result is the large difference between the $\Delta m_{\text {Faraday }}$ and the $\Delta m_{E Q C M}$ that can be observed in aqueous electrolyte, but in propylene-based electrolyte as well (Figure S15) and of greater amplitude. In both cases $\Delta m_{E Q C M}$ is substantially higher than $\Delta m_{\text {Faraday }}$ and this cannot be ascribed to the evolution upon cycling of the hydrodynamic or viscoelastic property of the film as every parameters have previously been verified. Tsionsky and co-workers proposed the formation of a "viscous layer" at the flat electrode/electrolyte interface. ${ }^{44}$ This increase of the local viscosity compared to the bulk of the electrolyte is due to the accumulation of cations in the double layer region when the electrode is negatively polarized and may contribute to frequency measurements. However, this contribution can partly explain the phenomenon and is negligible in comparison with this recorded mass difference. Since the incorporation of solvent molecules into $\mathrm{LiFePO}_{4}$ is impossible from structural point of view, we hypothesize that the solvent molecules could play a key role at the LFP-electrolyte interface. To elucidate the contribution of the solvent, the data was processed for scan rates for which the full delithiation is possible in both electrolytes (i.e. for aqueous $1 \mathrm{mV} / \mathrm{s}$ and organic $0.1 \mathrm{mV} / \mathrm{s}$ ). Supposing the involved complex $[\mathrm{Li}, \mathrm{z} \text { Solvent }]^{+}$, the number of solvent molecules associated to the $\mathrm{Li}^{+}$at the $\mathrm{EEI}$ can be determined thanks to the following equation:

$$
z=\frac{n_{\text {Solvent }}}{n_{L i^{+}}}=\frac{M_{L i}{ }^{+}}{M_{\text {Solvent }}} \times \frac{m-m_{L i^{+}}}{m_{L i^{+}}}
$$

where $n_{i}$ and $M_{i}$ are the number of moles and the molar mass, respectively, of the lithium or the solvent molecules (water or propylene carbonate). The entities, $m$ and $m_{L i}{ }^{+}$are the experimental mass and the electrochemical mass (calculated from the Faraday's law, Equation S2), respectively. 

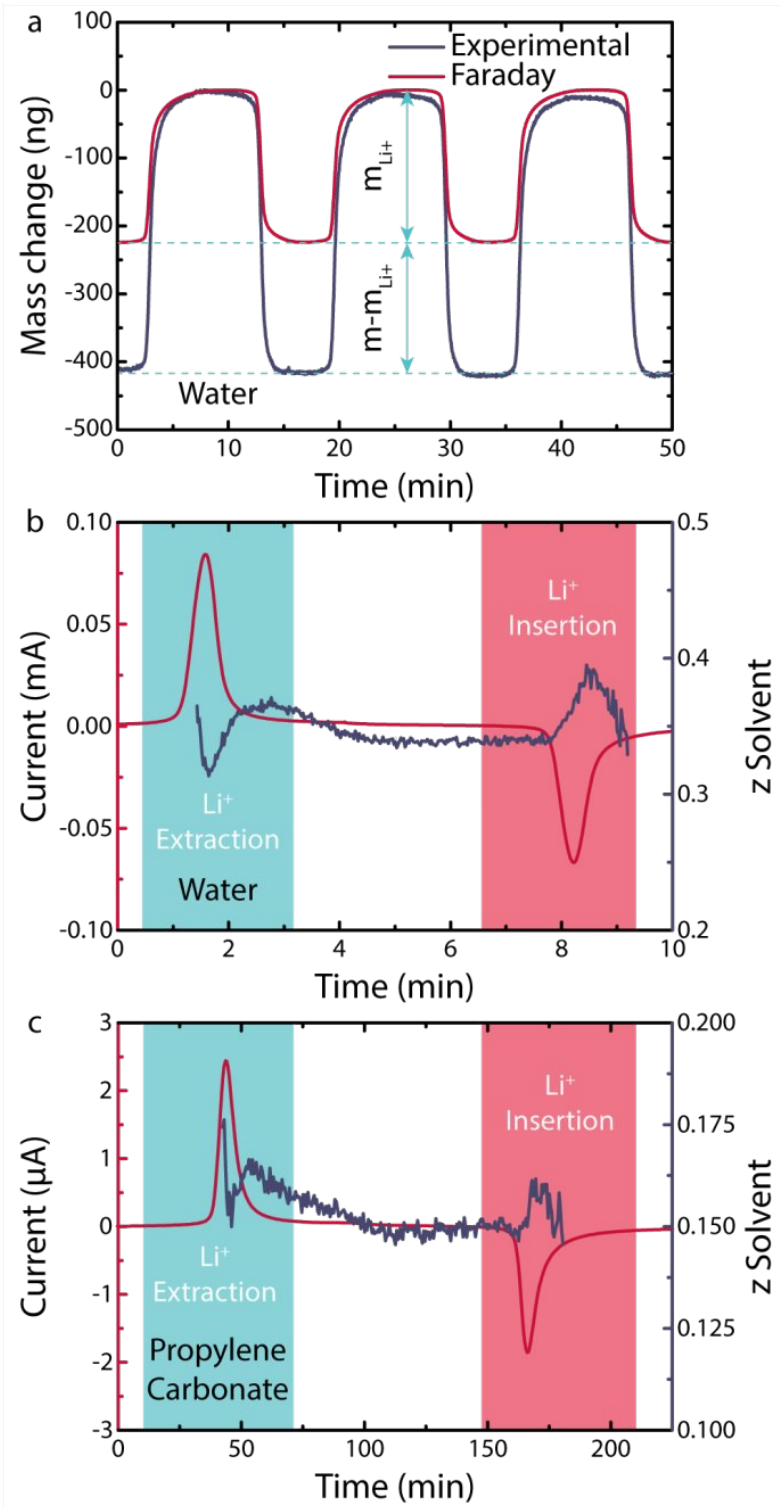

Figure 6: (a) Experimental ( $\Delta \mathrm{m}_{\mathrm{EQ}}$ ) $)$ and electrochemical mass comparison ( $\left.\Delta \mathrm{m}_{\mathrm{Faraday}}\right), C V$ at $1 \mathrm{mV} / \mathrm{s}$ in water. Determination of the number of solvents involved in the lithium insertion/extraction process (b) in $\mathrm{CV}$ at $1 \mathrm{mV} / \mathrm{s}$ in water and (c) in $\mathrm{CV}$ at $0.1 \mathrm{mV} / \mathrm{s}$ in propylene carbonate.

The results of these calculations are plotted in Figure $6 \mathrm{~b}$ and Figure $6 \mathrm{c}$ for $\mathrm{H}_{2} \mathrm{O}$-based and PC-based electrolytes, respectively. The number of solvent molecules involved in the lithium insertion/extraction process of $\mathrm{LiFePO}_{4}$ in water media (Figure 6b) averages at 0.35 with a decrease during the extraction and an increase during insertion. This value is lower and equal to 0.15 in propylene carbonate-based electrolyte (Figure $6 \mathrm{c}$ ) with however the same "up and down" feature during lithium insertion and extraction. Altogether, these results provide experimental evidence for the logical depletion and accumulation of solvent molecules at the EEI when lithium ions are extracted and inserted, respectively, with the amplitude of this feature been solvent dependent and the most pronounced in aqueous media. 


\section{Discussion and conclusion}

We have reported a comprehensive new protocol that combines a multi-step coating process, the design of a new hardware and verification steps to securely interpret the EQCM results into exploitable mass changes, hence facilitating the ingress of this user-friendly characterization tool in battery research. Regarding our coating process, it includes multi-steps offering reproducible film deposits with a lateral and vertical homogeneity that can be tuned depending on the characteristics of the active material and/or binder. Other techniques, enlisting physical vapor deposition ${ }^{8,9}$, electrodeposition ${ }^{16,18,45}$ and sol-gel processes ${ }^{13-15,17}$ offer the benefit of fabricating thin, rigid, dense and fairly smooth surface layer on the QCM resonator. However, they are limited to a few battery materials due to the limited temperature range of metal coated-quartz resonator stability $\left(<300{ }^{\circ} \mathrm{C}\right)$. Therefore, an important asset of the spray-coating based deposition process is its feasible implementation to various active materials that can be synthetized beforehand and deposited under "soft conditions" without any perturbation to the resonator properties. If necessary, there are options for reaching higher electrode preparation temperatures by working with $\mathrm{GaPO}_{4}$ as resonator material with the possibility of reaching up to $600^{\circ} \mathrm{C} .46$

For proof of concept, we have used the well-known $\mathrm{LiFePO}_{4}$ phase as a model material and the quality of the films obtained for gravimetric measurements was secured via QCM-D measurements at multiharmonic that successfully revealed the hydrodynamic behavior of a flat surface film, rigidly attached to the resonator in both aqueous and non-aqueous electrolytes. We should recall that spray coating was firstly introduced by Levi et al. ${ }^{43}$ in 2009 to characterize the ionic fluxes in microporous carbons as supercapacitor electrodes. However, the procedure was succinctly described, and in certain cases leads to non-continuous coatings, depending on the active materials particle size and the nature of the binder. ${ }^{31}$ Recently, a vacuum filtration-and-transfer (VFT) technique was described for the same purpose $^{47}$ with in this case the active material been filtered through a cellulose membrane then transferred to surface of the resonator by dissolving the cellulose with acetone. According to the authors this method overqualified spray drying for obtaining homogeneous coatings with relative ease and reproducibility. However, this protocol do not permit the use of common binders of the battery field and especially PVDF, which are soluble in polar solvent as is the case for cellulose. Moreover, the presence of suitable binder is an important factor to obtain rigidly attached films at the surface of the QCM resonator. ${ }^{28}$ Such a brief literature survey together with our own work strongly conveys that there is not a single ideal deposit technique because of the different physical properties of the active materials studied.

Most of the publications employing EQCM in organic media, describe measurements performed in a glovebox or restricted to one brand of frequency read-out. To alleviate these burdens we have developed a new cell dedicated to EQCM measurement for battery and supercapacitor testing. It offers several advantages: mimic the battery configuration, ensure airtightness (no exposure to any contaminant) while having low impedance and being reproducible. Moreover, besides its versatility of hosting either aqueous or non-aqueous electrolyte, it is compatible with the complementary techniques comprised in the QCM toolbox: EQCM, EQCM-R, network analyzer and EQCM-D. However, for long testing periods aiming to track the SEI dynamics in battery materials we recommend to conduct EQCM measurements in a temperature controlled atmosphere to obtain stable response over time. Additionally, the practicality of our approach, mainly for analyzing active materials that can suffer from the larger constraints during cycling is rooted in the dual monitoring of motional resistance and frequency. Hence, our suggestion is of using EQCM-R as a convenient qualitative viscoelastic indicator, which can be completed by EQCM-D if viscoelasticity is predominant. Let us reiterate that a small variation of the motional resistance during cycling attests that the rigidity of the composite LFP films 
is preserved during (de)insertion of $\mathrm{Li}^{+}$. Checking these properties is essential to avoid a large dissipation of energy when modified QCM resonators are immersed in an electrolyte, which may result in an over/under estimation of the real mass change. This is also mandatory to obtain valuable new insights on the insertion/extraction mechanism of guest ions into framework structures, provided by having a robust and stable over time binder-active material composite.

New trends in the battery field, such as the use of aqueous electrolytes as alternatives to organic counterparts, brings the necessity to revisit even the model materials behavior, under the new operation conditions, hence the ever-increasing interest for the EQCM technique. For example, the higher rate capability of LFP particles in aqueous electrolytes compared with organic counterparts, even in practical batteries ${ }^{48}$, has been demonstrated but underlying reasons are poorly understood. Addressing this question by our EQCM-R based strategy led us to directly assess the partial or total LFP delithiation conditions, depending on the potential scan rate, in two different media. We unambiguously proved that the $\mathrm{Li}^{+}$insertion/extraction mechanism in $\mathrm{LiFePO}_{4}$ is solvent-dependent. Moreover, by comparing the experimental $\Delta m_{E Q C M}$ and theoretical $\Delta m_{\text {Faraday }}$ mass changes accompanying the insertions and extraction of $\mathrm{Li}^{+}$ions in both electrolytes we provide evidence for a solvent-assisted insertion process with the number of solvent molecules pertaining to the solvation shell being markedly greater for water than for propylene carbonate-based electrolytes. This higher concentration variation of water molecules at the EEI of composite LFP electrode enables a faster insertion/extraction at the vicinity of the active material particle. Song et al. ${ }^{49}$ reported similar conclusions based on EQCM studies performed with binder-free coatings. However, they solely obtained an approximate estimation of the number of solvent molecules involved the interfacial mechanism, because of gravimetric curves presenting unexpected profiles. We believe that the reason is rooted in the absence of binder in their coatings, hence re-insisting that binders are essential to accommodate the strain caused by the $\mathrm{Li}^{+}$insertion in LFP, avoiding its viscoelastic evolution. ${ }^{25,26}$

Such findings are independently supported by $a b$ initio calculations ${ }^{50}$ that show the formation of a chemisorbed $\mathrm{H}_{2} \mathrm{O}$ molecules at the interface with $\mathrm{LiFePO}_{4}$ which facilitates the lithium desolvation process near the surface and therefore accelerates the Li-ion transport across the EEI. This scenario do not occur in presence of big organic molecules alike propylene carbonate owing to steric hindrance. These calculations, combined with our findings regarding the depletion/increase of the solvent concentration during the lithium insertion/extraction demonstrate the lithium desolvation from its water shell near the active material surface while in organic media the removal of the organic solvation shell happens further apart from the interface. This implies a higher energy of desolvation in nonaqueous rather than in aqueous electrolyte, hence accounting for the solvent-dependent rate capability trends observed with LFP.

Although presently limited to $\mathrm{LiFePO}_{4}$, our developments of the EQCM tooling for battery research together with means of getting insights on the behavior of solvated $\mathrm{Li}^{+}$ions at $\mathrm{EEI}$ can be extended to other charge carriers $\left(\mathrm{Na}^{+}, \mathrm{K}^{+}, \ldots\right)$ and electrode materials, in view of screening/predicting in which electrolyte composition, optimum performance can be reached. Additionally, the strategy can be implemented to study the evolution of electrodes during cycling, such as distinguishing a viscoelastic SEl layer formed on a rigid electrode, using motional resistance monitoring as an indicator. Moreover, the proposed EQCM-R bridges the gap between classical EQCM and complex multiharmonic EQCM-D. EQCM-R measurements offer a versatile and friendly method, which is compatible with today battery tooling via the design of a new cell hardware. It opens a few opportunities to better quantify interfacial insertion kinetics of intervening species (e.g. $\mathrm{Li}^{+}$and solvent molecules) by performing EIS coupled measurements (the so-called ac-electrogravimetry) of battery electrodes as is being initiating in our group. Altogether, we hope this work will help in a wider acceptance of EQCM techniques by the 
battery community to study a wide panel of active electrode materials but also a broad variety of Electrode-Electrolyte Interface phenomena such as ion solvation and transport for instance. 


\section{References}

(1) Tarascon, J.-M.; Armand, M. Nature 2001, 414 (6861), 359-367.

(2) Berg, E. J.; Villevieille, C.; Streich, D.; Trabesinger, S.; Novák, P. J. Electrochem. Soc. 2015, 162 (14), A2468.

(3) Zhang, X.; van Hulzen, M.; Singh, D. P.; Brownrigg, A.; Wright, J. P.; van Dijk, N. H.; Wagemaker, M. Nat. Commun. 2015, 6 (1), 1-7.

(4) Yamamoto, K.; Minato, T.; Mori, S.; Takamatsu, D.; Orikasa, Y.; Tanida, H.; Nakanishi, K.; Murayama, H.; Masese, T.; Mori, T.; Arai, H.; Koyama, Y.; Ogumi, Z.; Uchimoto, Y. J. Phys. Chem. C 2014, 118 (18), 9538-9543.

(5) Peled, E.; Menkin, S. J. Electrochem. Soc. 2017, 164 (7), A1703-A1719.

(6) Sauerbrey, G. Z. Für Phys. Hadrons Nucl. 1959, 155 (2), 206-222.

(7) Aurbach, D.; Zaban, A. J. Electroanal. Chem. 1995, 393 (1-2), 43-53.

(8) Kwon, K.; Kong, F.; McLarnon, F.; Evans, J. W. J. Electrochem. Soc. 2003, 150 (2), A229-A233.

(9) Kwon, K.; Evans, J. W. Electrochimica Acta 2004, 49 (6), 867-872.

(10) Li, J.-T.; Chen, S.-R.; Fan, X.-Y.; Huang, L.; Sun, S.-G. Langmuir 2007, 23 (26), 13174-13180.

(11) Liu, T.; Lin, L.; Bi, X.; Tian, L.; Yang, K.; Liu, J.; Li, M.; Chen, Z.; Lu, J.; Amine, K.; Xu, K.; Pan, F. Nat. Nanotechnol. 2019, 14 (1), 50-56.

(12) Dubouis, N.; Lemaire, P.; Mirvaux, B.; Salager, E.; Deschamps, M.; Grimaud, A. Energy Environ. Sci. 2018, 11 (12), 3491-3499.

(13) Yang, Y.; Shu, D.; Yu, H.; Xia, X.; Lin, Z. G. J. Power Sources 1997, 65 (1-2), 227-230.

(14) Shouji, E.; A. Buttry, D. Electrochimica Acta 2000, 45 (22), 3757-3764.

(15) Bueno, P. R.; Faria, R. C.; Avellaneda, C. O.; Leite, E. R.; Bulhões, L. O. S. Solid State Ion. 2003, 158 (3), 415-426.

(16) Razzaghi, F.; Debiemme-Chouvy, C.; Pillier, F.; Perrot, H.; Sel, O. Phys. Chem. Chem. Phys. 2015, 17 (22), 14773-14787.

(17) Bueno, P. R.; Faria, R. C.; Bulhões, L. O. S. Solid State Ion. 2005, 176 (11-12), 1175-1180.

(18) Song, J.; Noked, M.; Gillette, E.; Duay, J.; Rubloff, G.; Lee, S. B. Phys. Chem. Chem. Phys. 2015, 17 (7), 5256-5264.

(19) Lemaire, P.; Sel, O.; Alves Dalla Corte, D.; ladecola, A.; Perrot, H.; Tarascon, J.-M. ACS Appl. Mater. Interfaces 2020, 12 (4), 4510-4519.

(20) Levi, M. D.; Shpigel, N.; Sigalov, S.; Dargel, V.; Daikhin, L.; Aurbach, D. Electrochimica Acta 2017, 232, 271-284.

(21) Yang, Z.; Dixon, M. C.; Erck, R. A.; Trahey, L. ACS Appl. Mater. Interfaces 2015, 7 (48), 2658526594.

(22) Dargel, V.; Shpigel, N.; Sigalov, S.; Nayak, P.; Levi, M. D.; Daikhin, L.; Aurbach, D. Nat. Commun. 2017, 8 (1).

(23) Kitz, P. G.; Lacey, M. J.; Novák, P.; Berg, E. J. Anal. Chem. 2019, 91 (3), 2296-2303.

(24) Kitz, P. G.; Novák, P.; Berg, E. J. ACS Appl. Mater. Interfaces 2020, 12 (13), 15934-15942.

(25) Shpigel, N.; Sigalov, S.; Levi, M. D.; Mathis, T.; Daikhin, L.; Janes, A.; Lust, E.; Gogotsi, Y.; Aurbach, D. Joule 2018, 2 (5), 988-1003.

(26) Shpigel, N.; Levi, M. D.; Cheng, X.; Cao, T.; Wu, R.; Mathis, T. S.; Zhang, Y.; Aurbach, D.; Gogotsi, Y. ACS Energy Lett. 2019, 1907-1917.

(27) Shpigel, N.; Levi, M. D.; Sigalov, S.; Girshevitz, O.; Aurbach, D.; Daikhin, L.; Jäckel, N.; Presser, V. Angew. Chem. Int. Ed. 2015, 54 (42), 12353-12356.

(28) Dargel, V.; Jäckel, N.; Shpigel, N.; Sigalov, S.; Levi, M. D.; Daikhin, L.; Presser, V.; Aurbach, D. ACS Appl. Mater. Interfaces 2017, 9 (33), 27664-27675.

(29) Dargel, V.; Levi, M. D.; Daikhin, L.; Aurbach, D. Russ. J. Electrochem. 2017, 53 (9), 980-993.

(30) Levi, M. D.; Daikhin, L.; Aurbach, D.; Presser, V. Electrochem. Commun. 2016, 67, $16-21$.

(31) Shpigel, N.; Levi, M. D.; Aurbach, D. Energy Storage Mater. 2019, 21, 399-413.

(32) Johannsmann, D. The Quartz Crystal Microbalance in Soft Matter Research: Fundamentals and Modeling; Soft and Biological Matter; Springer International Publishing, 2015. 
(33) Montagut, Y. J.; García, J. V.; Jiménez, Y.; March, C.; Montoya, A.; Arnau, A. Rev. Sci. Instrum. 2011, 82 (6), 064702.

(34) Yunker, P. J.; Still, T.; Lohr, M. A.; Yodh, A. G. Nature 2011, 476 (7360), 308-311.

(35) Barbosa, J. C.; Dias, J. P.; Lanceros-Méndez, S.; Costa, C. M. Membranes 2018, 8 (3), 45.

(36) Cambon, O.; Haines, J.; Fraysse, G.; Keen, D. A.; Tucker, M. G. J. Phys. IV Proc. 2005, 126, 2730.

(37) Goubaa, H.; Escobar-Teran, F.; Ressam, I.; Gao, W.; El Kadib, A.; Lucas, I. T.; Raihane, M.; Lahcini, M.; Perrot, H.; Sel, O. J. Phys. Chem. C 2017, 121 (17), 9370-9380.

(38) O'sullivan, C. K.; Guilbault, G. G. Biosens. Bioelectron. 1999, 14 (8), 663-670.

(39) Agrisuelas, J.; Gabrielli, C.; García-Jareño, J. J.; Perrot, H.; Sel, O.; Vicente, F. Electrochimica Acta 2015, 176, 1454-1463.

(40) Kanazawa, K. K.; Gordon, J. G. Anal. Chim. Acta 1985, 175, 99-105.

(41) Shpigel, N.; Levi, M. D.; Sigalov, S.; Daikhin, L.; Aurbach, D. Acc. Chem. Res. 2018, 51 (1), 6979.

(42) Aurbach, D.; Markovsky, B.; Salitra, G.; Markevich, E.; Talyossef, Y.; Koltypin, M.; Nazar, L.; Ellis, B.; Kovacheva, D. J. Power Sources 2007, 165 (2), 491-499.

(43) Levi, M. D.; Salitra, G.; Levy, N.; Aurbach, D.; Maier, J. Nat. Mater. 2009, 8 (11), 872-875.

(44) Tsionsky, V.; Daikhin, L.; Gileadi, E. J. Electrochem. Soc. 1995, 142 (12), L233-L234.

(45) Kim, L. T. T.; Gabrielli, C.; Perrot, H.; Garcia-Jareno, J.; Vicente, F. Electrochimica Acta 2012, 84, 35-48.

(46) Jakab, S.; Picart, S.; Tribollet, B.; Rousseau, P.; Perrot, H.; Gabrielli, C. Anal. Chem. 2009, 81 (13), 5139-5145.

(47) Zhang, Q.; Levi, M. D.; Chai, Y.; Zhang, X.; Xiao, D.; Dou, Q.; Ma, P.; Ji, H.; Yan, X. Small Methods 2019, 3 (11), 1900246.

(48) Zeng, X.; Liu, Q.; Chen, M.; Leng, L.; Shu, T.; Du, L.; Song, H.; Liao, S. Electrochimica Acta 2015, $177,277-282$.

(49) Song, X.; Liu, T.; Amine, J.; Duan, Y.; Zheng, J.; Lin, Y.; Pan, F. Nano Energy 2017, 37, 90-97.

(50) Zheng, J.; Hou, Y.; Duan, Y.; Song, X.; Wei, Y.; Liu, T.; Hu, J.; Guo, H.; Zhuo, Z.; Liu, L.; Chang, Z.; Wang, X.; Zherebetskyy, D.; Fang, Y.; Lin, Y.; Xu, K.; Wang, L.-W.; Wu, Y.; Pan, F. Nano Lett. 2015, 15 (9), 6102-6109.

\section{Acknowledgments}

J.-M.T. acknowledges funding from the European Research Council (ERC) (FP/2014)/ERC Grant-Project 670116-ARPEMA. FEGSEM \& EDX instrumentation was hosted at the Institut des Matériaux de Paris Centre (IMPCFR2482) and was funded by Sorbonne Université, CNRS and by the C'Nano projects of the Région lle-de-France. 


\section{For Table of Contents Only}

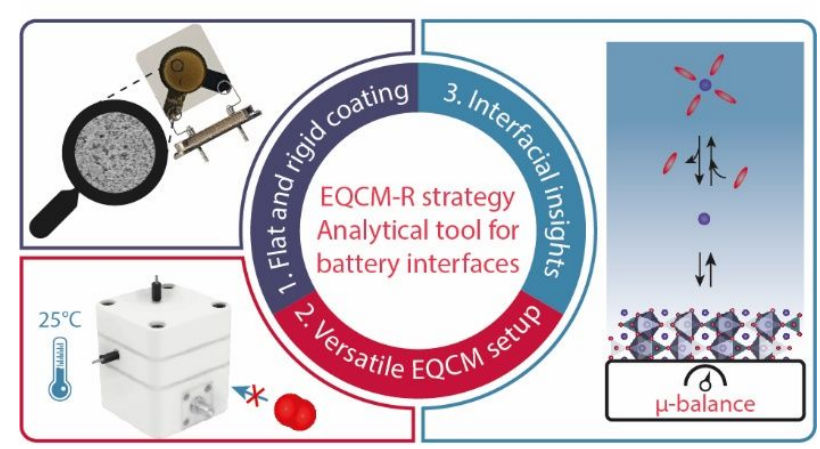

20

21 